\title{
Nanometer-scale GaAs clusters from organometallic precursors
}

\author{
Peter C. Sercel, Winston A. Saunders, Harry A. Atwater, \\ and Kerry J. Vahala \\ Department of Applied Physics, California Institute of Technology, Pasadena, California 91125 \\ Richard C. Flagan \\ Department of Chemical Engineering, California Institute of Technology, Pasadena, California 91125
}

(Received 23 March 1992; accepted for publication 26 May 1992)

\begin{abstract}
We report the synthesis of crystalline nanometer-scale GaAs clusters by homogeneous vapor-phase nucleation from organometallic precursors. Cluster synthesis is performed in a hot wall organometallic vapor-phase epitaxy reactor at atmospheric pressure. High resolution transmission electron microscopy studies reveal that the aerosol produced is composed of highly faceted single crystal GaAs particles in the $10-20 \mathrm{~nm}$ range. The influence of growth temperature and reactant concentration on cluster morphology is discussed.
\end{abstract}

We report the first direct observation of gas-phase homogeneous nucleation of GaAs by the thermophoretic collection of an aerosol of nanometer-scale microcrystallite GaAs clusters in an atmospheric organometallic vaporphase epitaxy (OMVPE) reactor. In addition to shedding light on a fundamental question about the reaction kinetics involved in OMVPE, this is the first report of gas-phase synthesis of nanometer-scale III/ $\mathrm{V}$ clusters from volatile precursor molecules. We have determined conditions under which nearly defect free, $10-20 \mathrm{~nm}$ scale GaAs microcrystallites form. These particles are in the quantum size regime which suggests that the process may be used to form optically active quantum dots. ${ }^{1}$ Thus gas-phase homogeneous nucleation in OMVPE, which has been regarded until now as a troublesome parasitic reaction, ${ }^{2,3}$ may ultimately form the basis of an aerosol technology for the fabrication of novel optoelectronic devices such as a quantum dot laser. ${ }^{4}$

The reactor used in these experiments is depicted in Fig. 1. A $34 \mathrm{~mm}$ diam, $700 \mathrm{~mm}$ long quartz process tube is mounted in a multizone furnace. Trimethyl gallium (TMG) was introduced by bubbling hydrogen through a liquid source held at $-10^{\circ} \mathrm{C}$. Heated injectors $(\mathrm{Z1}, \mathrm{Z} 2)$ permit TMG and $10 \% \mathrm{AsH}_{3}$ in hydrogen to be introduced into the process tube separately, allowing for the possibility of precracking the precursor molecules. Alternatively, the reactants can be mixed prior to injection by rerouting the arsine flow as shown in Fig. 1. The nucleation zone, Z3, is $18 \mathrm{~cm}$ long. Aerosol particles are collected thermophoretically on a holey carbon film which is mounted on a stainless steel fixture aligned coaxially in the collection zone, $\mathrm{Z5}$, of the process tube (Fig. 1). Z5 is held at a temperature of $350^{\circ} \mathrm{C}$ in all experiments to prevent the condensation of arsenic on the collection apparatus. Hydrogen, purified with a resin filter, is used as the carrier gas. The composition of the gas exiting the process tube is monitored with a quadrupole mass spectrometer (QMS).

In a typical experiment the TMG and $\mathrm{AsH}_{3}$ are preheated to $400^{\circ} \mathrm{C}$ in $\mathrm{Z} 1$ and $\mathrm{Z} 2$, respectively, before being introduced into the reaction zone. At this temperature negligible pyrolysis of the TMG or $\mathrm{AsH}_{3}$ occurs as previously determined by infrared absorption spectroscopy and mass spectroscopic analysis. ${ }^{5,6}$ This is verified by negligible de- creases in the arsine partial pressure and no $\mathrm{CH}_{4}$ partial pressure detectable on the QMS. The total flow is 2 SLM, with the partial pressures of TMG and arsine typically $1.25 \times 10^{-4}$ and $6.25 \times 10^{-3} \mathrm{~atm}$, respectively. For these flow conditions, the residence time in the reaction zone $(\mathrm{Z} 3)$ is $5 \mathrm{~s}$.

When the temperature in the nucleation zone $(Z 3)$ is held at $700^{\circ} \mathrm{C}$, solid $\mathrm{GaAs}$ deposits on the reactor walls within the first $10 \mathrm{~cm}$ of the nucleation zone, indicating that the TMG is entirely consumed within approximately $3 \mathrm{~s}$ of entering Z3. Downstream of this point, in Z5, a semitransparent yellow deposit is observed on the walls of the reactor tube and on the collection fixture. A transmission electron microscopy (TEM) micrograph of the collected deposit reveals it to consist of individual GaAs microcrystallites and some chainlike agglomerates (Fig. 2). The electron diffraction pattern of the particles, shown in the inset of Fig. 2, indexes to cubic zincblende structure, as shown in Fig. 3.

Figure 4 shows a high resolution transmission electron micrograph (HRTEM) of an agglomerate of smaller primary particles. In practice, HRTEM is easier to perform on the agglomerated clusters than on individual particles, owing to the fact that the agglomerates are better able to dissipate excess charge and heat induced by the electron beam than are individual particles. Within several of the clusters shown, lattice fringes and faceting indicative of 〈111) bounding planes are clearly evident. Some of the crystalline faces have as few as 30 atomic rows. The lattice fringes on the particles extend to within $1 \mathrm{~nm}$ of the cluster surface, consistent with the formation of an amorphous oxide layer on the particles on exposure to air.

We have investigated the role of growth temperature and reactant concentration on the nucleation and morphology of the clusters. By analyzing low magnification micrographs, such as Fig. 2, we have measured the size distributions of the primary particles for runs at different growth temperatures in the nucleation zone ( $\mathrm{Z3})$. We find that the mean particle diameter is strongly infiuenced by the growth temperature. By fitting the measured particle size distributions to a log-normal distribution, a median particle diameter $\bar{D}_{\mathrm{pg}}$ and geometric standard deviation $\sigma_{g}$ for the distribution are found. Geometric standard deviations 


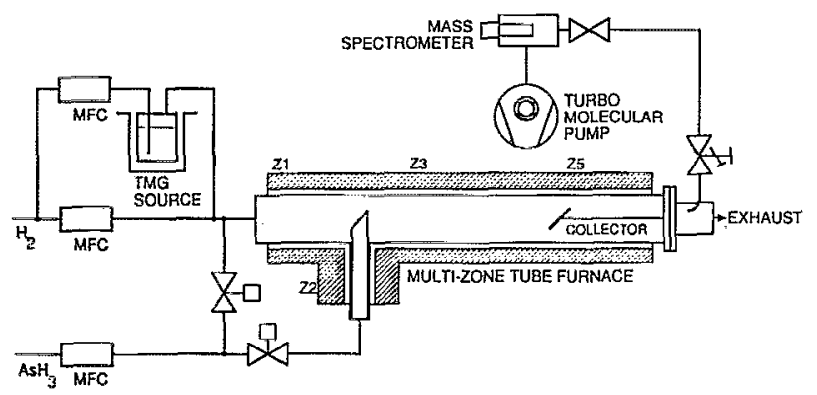

FIG. 1. Schematic of the experimental apparatus for gas-phase synthesis of nanometer-scale GaAs clusters. The group III precursor (TMG) is introduced into the hot wall reactor by bubbling $\mathrm{H}_{2}$ carrier gas through a liquid source. The group $\mathrm{V}$ precursor, $\mathrm{AsH}_{3}$, can be mixed with the TMG outside the furnace (at room temperature) or inside the furnace (at elevated temperature). All flows are regulated by mass flow controllers (MFCs). The process runs at atmospheric pressure. A quadrupole mass spectrometer, connected to the exhaust of the process tube by a sampling line, is used to monitor reactant concentrations.

are in the range $\sigma_{g}=1.2-1.3$. A typical data set and its fit are shown in Fig. 5(a). Figure 5(b) shows the behavior of the median crystallite diameter and $\sigma_{g}$ as a function of reciprocal temperature. As the temperature in the nucleation zone $(\mathrm{Z} 3)$ is increased from $600^{\circ} \mathrm{C}(1000 / T=1.14$ $\left.\mathrm{K}^{-1}\right)$ to $800{ }^{\circ} \mathrm{C}\left(1000 / T=0.93 \mathrm{~K}^{-1}\right)$, the median particle diameter rises continuously from 12 to $19 \mathrm{~nm}$. Doubling the input TMG and $\mathrm{AsH}_{3}$ concentrations relative to the conditions of Figs. 2 and 3, the mean particle diameter is increased by approximately $10 \%$ with no noticeable

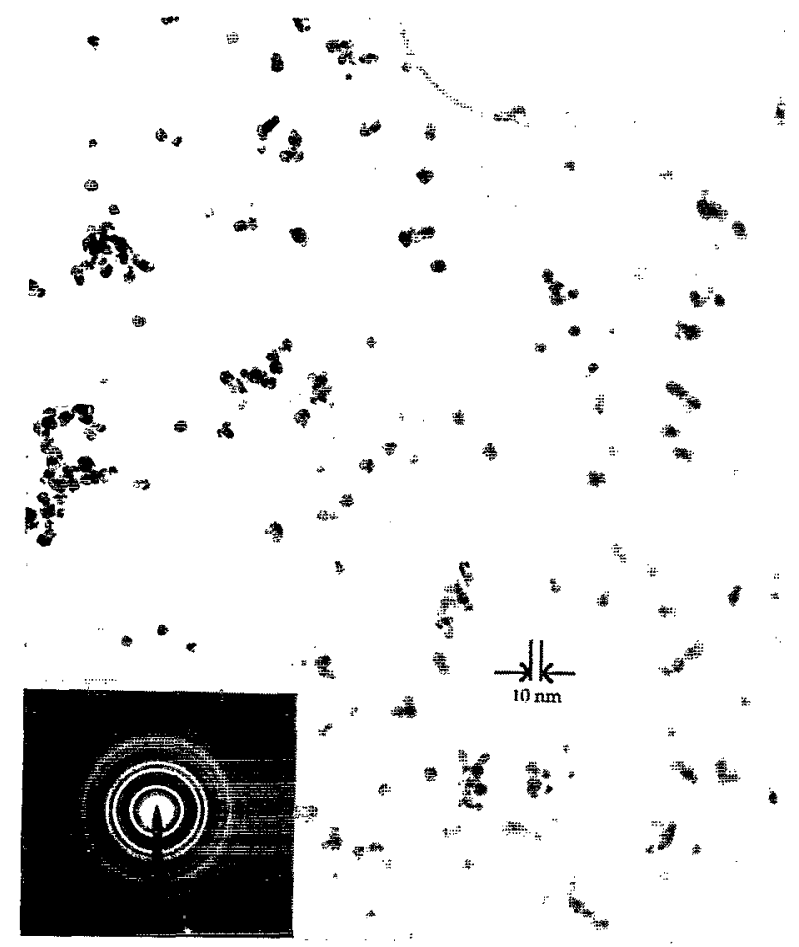

FIG. 2. Bright field transmission electron micrograph of GaAs clusters collected on a holey carbon substrate. The temperature in $\mathrm{Z3}$ was $700^{\circ} \mathrm{C}$, the partial pressure of TMG was $1.25 \times 10^{-4} \mathrm{~atm}$, the V/III ratio was 50 , and the total flow was 2 SLM. Inset: electron diffraction pattern of the clusters. The pattern indexes to zincblende (see Fig. 3).

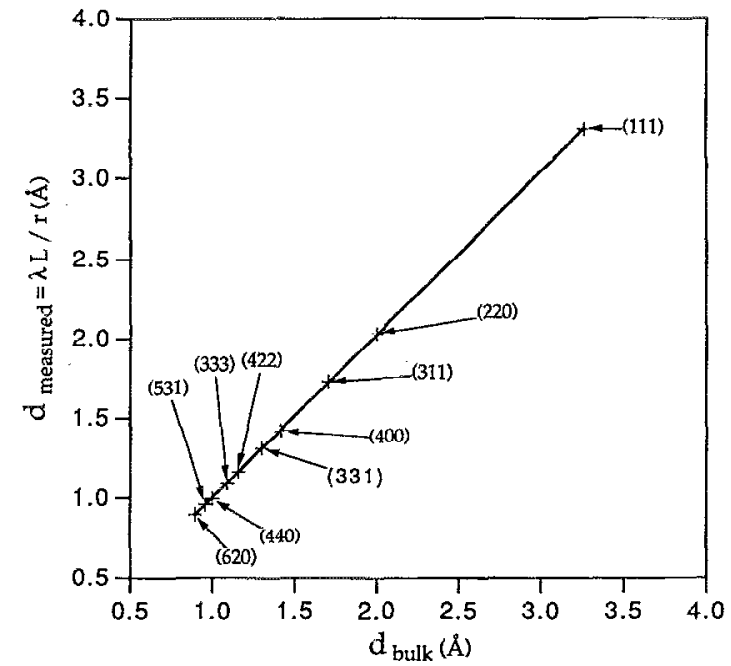

FIG. 3. Interplanar spacings for GaAs clusters shown in Fig. 2 vs bulk interplanar spacings. Measured values correspond to the ring radii $R$ according to the equation $d_{\text {measured }}=\lambda L / R$, where $\lambda L$ is the camera constant. The lattice constant of the GaAs clusters is equal to the bulk value to within experimental uncertainty $(5 \%)$.

change in particle morphology. Additionally, we have generated clusters by mixing TMG and arsine both at room temperature and at $400^{\circ} \mathrm{C}$ and find no difference in particle morphology.

Several important questions remain regarding the growth mechanism of the primary GaAs clusters. Gasphase infrared absorption measurements showing the presence of TMG- $\mathrm{AsH}_{3}$ adducts, ${ }^{5}$ suggest that GaAs may form homogeneously, nucleating (GaAs) ${ }_{n}$ clusters with $n$

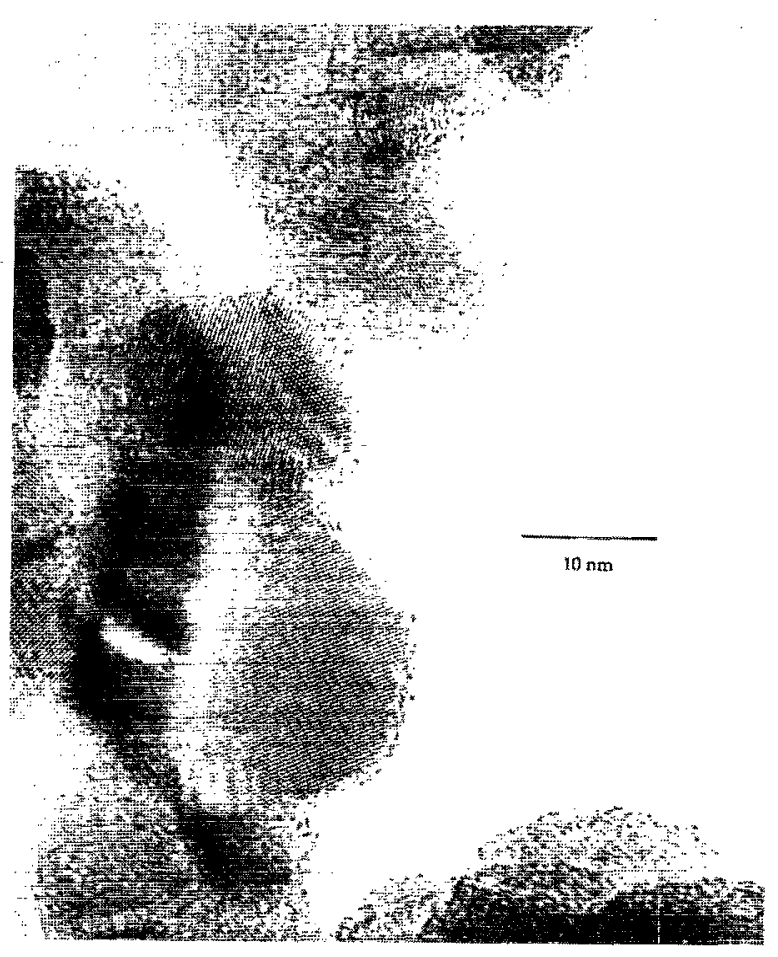

FIG. 4. High resolution transmission electron micrograph of a chain agglomerate produced under the same conditions as Fig. 2. 


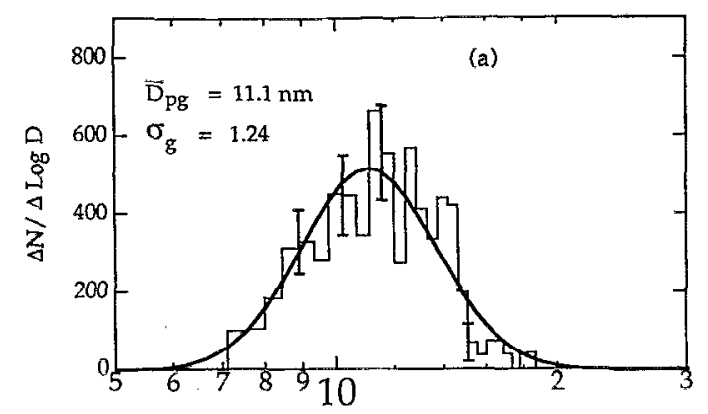

$\mathrm{D}(\mathrm{nm})$

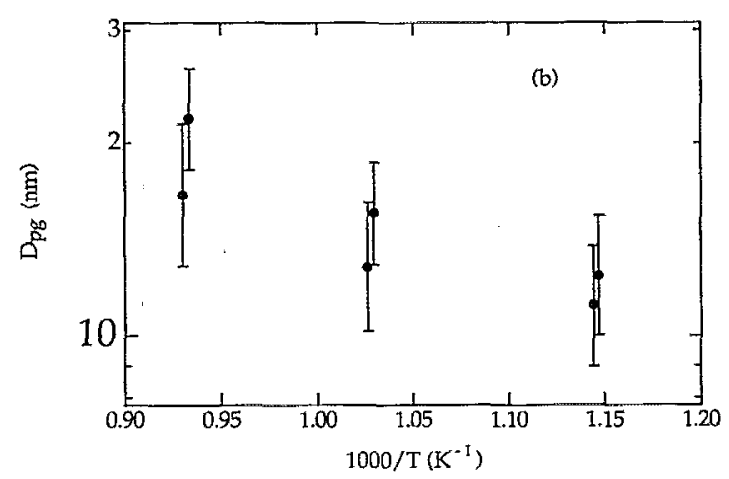

FIG. 5. Analysis of the size distribution of GaAs clusters produced at various temperatures. (a) Representative data for a run with growth temperature $600^{\circ} \mathrm{C}$, plotted as a histogram $\Delta N / \Delta \log D_{p}$ vs $D_{p}$, the particle diameter. The solid line is a best fit to a $\log$-normal distribution. (b) Semilog plot of median particle diameter $\bar{D}_{\mathrm{pg}}$ vs reciprocal temperature. The geometric standard deviation $\sigma_{g}$ determined for each point is represented on the plot as a vertical bar of height $2 \sigma_{g}$.

in the range $10-20 .^{7}$ These clusters may then increase to the $10 \mathrm{~nm}$ size range by coagulation. Subsequent grain boundary migration through the sintered particle may consolidate the agglomerate into the single crystal, faceted clusters seen in Figs. 2 and 3. Alternatively, further growth to the observed sizes in the $10 \mathrm{~nm}$ range may proceed by epitaxial deposition from the supersaturated vapor phase. Since the microscopic crystal planes of the clusters are much smaller than surface diffusion lengths, adsorbed $\mathrm{Ga}$ and As atoms can diffuse to fast growing crystal planes, leaving the cluster bounded by slow growth planes as observed in Fig. $4 .^{8}$ With the present data, it is impossible to distinguish between these two mechanisms, although the particle size distributions are somewhat narrower than would be expected if coagulation were the dominant growth mechanism $\left(\sigma_{g} \approx 1.46\right) .{ }^{9}$ It is also possible that GaAs cluster growth occurs through a two step process by the reaction of nanometer-scale droplets of $\mathrm{Ga}$ or polymonomethyl gallium polymer with the $\mathrm{AsH}_{3}$ ambient. Experiments designed to distinguish among these possible mechanisms are the focus of continuing work.

The present aerosol technique has several significant advantages over previous methods for producing nanometer-scale GaAs clusters. For example, synthesis in quinoline solution produces GaAs cluster with significant impurities and unequal $\mathrm{Ga}$ and As abundances, ${ }^{10,11}$ while GaAs molecular beam epitaxy on silica substrates produces irregular particles in intimate contact with a foreign substrate. ${ }^{12}$ Synthesis of nanometer-scale GaAs clusters by homogeneous nucleation from an exploding wire vapor has been recently demonstrated. ${ }^{13}$ However, we feel that the process described here is more readily compatible with the incorporation of the GaAs clusters in a passivating medium of AlGaAs both in the aerosol phase ${ }^{14}$ and ultimately in a growing epitaxial structure.

This work was supported by NSF Grant No. CTS8912328 and the Powell Foundation. PCS acknowledges support by the ATT Foundation.

${ }^{1}$ L. E. Brus, J. Chem. Phys. 80, 4402 (1984).

${ }^{2}$ G. B. Stringfellow, J. Cryst. Growth 68, 111 (1984).

${ }_{3}^{3}$ M. R. Leys and H. Veenvliet, J. Cryst. Growth. 55, 145 (1981).

${ }^{4}$ K. J. Vahala, IEEE J. Quantum Electron QE-24, 523 (1988).

${ }^{5} \mathrm{~J}$. Nishizawa and T. Kurabayashi, J. Electrochem. Soc. 130, 413 (1983).

${ }^{6}$ M. Yoshida, H. Watanabe, and F. Uesugi, J. Electrochem. Soc. 132, 677 (1985).

${ }^{7}$ Y. Takahashi, T. Soga, S. Sakai, M. Umeno, and S. Hattori, Jpn. J. Appl. Phys. 23, 709 (1984).

${ }^{8}$ S. Hersee, E. Barbier, and R. Blondeau, J. Cryst. Growth 77, 310 (1986).

${ }^{9}$ J. D. Landgrebe and S. E. Pratsinis, Ind. Eng. Chem. Res. 28, 1474 (1989).

${ }^{10}$ M. A. Olshavsky, A. N. Goldstein, and A. P. Alivisatos, J. Am. Chem. Soc. 112, 9438 (1990).

${ }^{11}$ H. Uchida, C. J. Curtis, and A. Nozik, J. Phys. Chem. 95, 5382 (1991).

${ }^{12}$ C. J. Sandroff, J. P. Harbison, R. Ramesh, M. J. Andrejco, M. S. Hedge, D. M. Huang, C. C. Chang, and E. M. Vogel, Science 245, 391 (1989).

${ }^{13}$ W. A. Saunders, P. C. Sercel, H. A. Atwater, K. J. Vahala, and R. C. Flagan, Appl. Phys. Lett. 60, 950 (1992).

${ }^{14}$ W. A. Saunders, K. J. Vahala, H. A. Atwater, and R. C. Flagan, J. Appl. Phys. 71, 806 (1992). 